\title{
70 - Building the food pyramid since early infancy
}

\author{
A Vania' ${ }^{1}$, C Sorino ${ }^{2}$, C Totaro Fila ${ }^{3}$, E Bombelli $^{3}$ and C Cannella ${ }^{2}$
}

${ }^{1}$ Centro di Dietologia e Nutrizione Pediatrica, Italy: ${ }^{2}$ Istituto di Scienza dell'Alimentazione, Dip.to di Fisiopatologia Medica, Facolta di Medicina e Chirurgia, 'Sapienza' Universita di Roma, Italy: ${ }^{3}$ Mellin Italia S.p.A., Milano, Italy

Aim: To design some food pyramids (FP) useful to families and paediatricians since baby's first year of life, in order to improve infant's feeding culture and knowledge, and to help preventing obesity since early infancy. Introduction: Since 1978, many different FP have been produced all around the world. Presently, many different types of FP exist with special focus on specific people categories or age groups. However, childhood benefits of various and different $\mathrm{FP}$, infancy does not.

Method: Two different FP, based on Italian weaning culture and nutritionally accurate, were produced, to be used by mothers, care takers, paediatricians, at two steps of infant's life: when weaning is started, then when two meals are complementing breast-feeding. The FP are printed on small dimension (1/2-A4) sheets, in blocks to be delivered to specialists, who can give the first and second sheet to every family in two different moments (before the 6th, after the 7th month). Sheets' content is not branded in any way. Each FP, albeit graphically very clear, is completed with several explications: doses and quality of different foods, practical examples, etc. The two FP have both a daily and a weekly side, meant to be particularly useful for the second weaning step. A link to www.piramideitaliana.it (website with updated nutritional information and FP for both adults and children) completes the front-site. Sheet's back-site is partly preprinted for basic infant's data (age, weight, length, head circumference) and with extra-information about babyfoods in general, and partly blank, for paediatrician's suggestions/prescriptions.

Funding: The project related to this abstract was partially funded by Mellin Italia S.p.A.

\section{1 - In form: a project to fight adiposity in children and adolescents}

\author{
R Birnbacher ${ }^{1}$, E Ardelt-Gattinger ${ }^{2}$, S Ring-Dimitriou ${ }^{2}$ and K Brugger ${ }^{3}$ \\ ${ }^{1}$ Regional Hospital Villach, Austria: ${ }^{2}$ University of Salzburg, Austria: ${ }^{3}$ Sanicademia, Austria
}

Introduction: Depending on region and age, up to onethird of children and young adults are overweight or suffer from obesity. A local project in Villach is adopting an innovative and interdisciplinary method and combines this with a preventive approach in the meso-system.

Method: A team of people from various disciplines, e.g., doctors, psychologists, nutritionists, dietitians, sports scientists and pedagogues have been working with more than eighty-four children and adolescents between 5 and 17 years of age since spring 2008. For a period of 2 years, the children and adolescents were offered two exercise units per week, and alternating psychological counselling or dietary information, as well as cooking courses. To be able to offer individual concepts tailored to the needs and requirements of the individual children, the groups were formed based on the first medical examination, the interdisciplinary Ad-EVA test system and the first psychological consultation. In medical terms, children qualified for the project when they were in the top weight percentile. In the framework of the examination, all relevant personal and family data of the children were recorded, and any additionally required checks were carried out (including endocrinological checks, ultrasound, OGTT, etc. according to AGA guidelines). The trainers had received instruction and training in a specific obesity trainer workshop in 2007. Several events in schools and kindergardens, at the Teacher-Training College and in other areas of the meso- and macro-system were organised.

Results: Changes were evaluated when the first half of the project period was concluded. At that time, some 35\% of the children and parents still were with the programme. Pre- and post-medical data and results of a physical fitness test of twenty-two children of the sports programme were evaluated, as well as questionnaires dealing with psychology, motivation to do sports and 